\title{
Die Thymidin-Phosphorylierung bei verschiedenen Entwidklungsstadien von Bufo bufo L.
}

\author{
F. Duspiva und E. Hansen-Delkeskamp \\ Zoologisches Institut der Universität Heidelberg, Physiologischer Lehrstuhl \\ Herrn Professor Dr. med. F. Büchner zum 70. Geburtstag gewidmet \\ (Z. Naturforschg. 20 b, 582-587 [1965] ; eingegangen am 28. Dezember 1964)
}

\begin{abstract}
Der Aktivitätsverlauf der Thymidin-phosphorylierenden Kinasen in der Frühentwicklung unterscheidet sich deutlich von bisherigen Daten über Enzyme des intermediären Stoffwechsels bei Amphibien. Die Aktivität ist im unbefruchteten Ei sehr hoch, sinkt nach der Befruchtung und bleibt bis zum mittleren Blastulastadium auf einem mittleren Niveau. Während der Gastrulation und Neurulation nimmt die Aktivität parallel der Mitoserate ab und steigt beim Wachstumsbeginn der Keime im Larvenstadium beträchtlich an.
\end{abstract}

Seit einiger Zeit ist bekannt, daß in Säugetiergeweben mit hoher Mitoserate wie der regenerierenden Leber und den Tumoren die Aktivität der DNSsynthetisierenden Enzyme stark erhöht ist gegenüber solchen mit geringer Zellteilungsrate wie der normalen Leber ${ }^{1}$. Der Aktivität dieser Enzyme hat man eine wichtige Funktion in der Kontrolle der DNS-Synthese zugeschrieben. Es gehören hierzu die Thymidinmonophosphat (TMP) zu Thymidintriphosphat (TTP) phosphorylierenden Kinasen ${ }^{1-3}$, die DNS-Polymerase ${ }^{4,5}$ und die DNS-ase ${ }^{6,7}$. Ferner ist die Wirksamkeit der DNS als „primer" von entscheidender Bedeutung ${ }^{8}$. In der Frühentwicklung des Seeigels Strongylocentrotus purpuratus ist während der Furchung ebenfalls eine höhere Aktivität der DNS-synthetisierenden Enzyme als bei Stadien mit geringem Mitoseindex gefunden worden ${ }^{9}$. Es ist noch nicht sicher geklärt, ob die Kinasen und die Polymerase in Geweben mit niedriger Zellteilungsrate wie der normalen Leber in geringer Konzentration oder in inaktiver Form vorliegen. Es scheint aber, daß sowohl die Aktivität der Kinasen ${ }^{3}$ als auch die der Polymerase ${ }^{10}$ durch Hemmstoffe beeinflußt wird.

1 J. N. Davidson, in: Biological Structure and Function, Academic Press, London, New York 1961, p. 95.

2 R. Kielley, Cancer Res. 23, 801 [1963].

${ }^{3}$ R. Kielley, Biochem. biophysic. Res. Commun. 10, 249 [1963].

4 R. M. S. Smellie, H. M. Keir u. J. N. Davidson, Biochim. biophysica Acta [Amsterdam] 35, 389 [1959].

5 R. M. Behri u. W. C. Schneider, Biochim. biophysica Acta [Amsterdam] 68, 34 [1963].

${ }_{6}$ S. Brody, Nature [London] 182, 1386 [1958].

7 K. N. Sarkar, M. A. Mukundan u. A. Devi, Nature [London] 200, 1205 [1963].
In gleicher Weise wie die Aktivität der TMP. phosphorylierenden Kinasen in der Leber nach Hepatektomie ansteigt, nimmt auch die Aktivität der Thymidin (TdR)-Kinase zu, obwohl TdR kein natürliches Zwischenprodukt der TTP-Synthese ist 11,12 .

In der vorliegenden Arbeit wurde die Phosphorylierung von TdR bei verschiedenen Entwicklungsstadien von Bufo bufo untersucht. Der gefundene Aktivitätsverlauf wird in Zusammenhang mit morphogenetischen Prozessen der Frühentwicklung diskutiert.

\section{Material und Methoden}

Haltung der Kröten: Die im Frühjahr gesammelten Kröten befanden sich in einem kopulationsbereiten Zustand und wurden im Kühlschrank bei +4 bis $+7{ }^{\circ} \mathrm{C}$ aufbewahrt. Die Eiablage erfolgte spontan innerhalb von $24-48 \mathrm{Stdn}$, wenn Weibchen und Männchen bei Zimmertemperatur zusammengebracht wurden. Die Eier wurden in Glasschalen mit Tümpelwasser bei $17-21{ }^{\circ} \mathrm{C}$ kultiviert. Unbefruchtete Eier konnten leicht gewonnen werden,

8 E. M. Gogol u. E. Rosenberg, Biochem. biophysic. Res. Commun. 14, 565 [1964].

${ }^{9}$ R. T. Hinegardner u. D. Mazia, Science [Washington] 136, 326 [1962].

10 M. G. Burdon, R. M. S. Smellie u. J. N. Davidson, Biochim. biophysica Acta [Amsterdam] 91, 46 [1964].

11 S. M. Weissman, R. M. S. Smellie u. J. Paul, Biochim. biophysica Acta [Amsterdam] 45, 101 [1960].

12 E. D. Gray, S. M. Weissman, J. Richards, D. Bell, H. M. Keir, R. M. S. Smellie u. J. N. Davidson, Biochim. biophy. sica Acta [Amsterdam] 45, 111 [1960]. 
wenn den Weibchen 600 I.E. Primogonyl (Schering AG Berlin) in den dorsalen Lymphsack injiziert wurden. Die Weibchen laichten im Zeitraum von $24-48$ Stdn. nach der Injektion ab.

Folgende Entwicklungsstadien wurden untersucht:

Unbefruchtete Eier.

Befruchtete Eier: 30 - 45 min nach der Befruchtung. 4-Zellstadien: sofort nach Erreichen der Vierzelligkeit; ca. 2,5 Stdn. nach der Befruchtung.

Mittlere Blastulae: Oberfläche noch deutlich durch die Zellen gefurcht; ca. $12 \mathrm{Stdn}$. nach der Befruchtung.

Mittlere Gastrulae: Durchmesser der noch nicht invaginierten Dotterzellen etwa ein Drittel des Keimdurchmessers; ca. 33 Stdn. nach der Befruchtung.

Neurulae: Neuralfalten beginnen sich einander zu nähern; ca. 57 Stdn. nach der Befruchtung.

Schwanzknospenlarven: schlüpfreif; 88-96 Stdn. nach der Befruchtung.

Larven: $160-190$ Stdn. nach der Befruchtung.

Kinasetest: 80 Keime wurden nach Entfernen der Gallerte im Glashomogenisator nach P otter und Elvehjem mit soviel Homogenisationsmedium homogenisiert, daß das Endvolumen $2 \mathrm{ml}$ betrug. Das Medium enthielt 0,05-m. GlycylglycinPuffer $p_{\mathrm{H}} 7,8$ und $0,01-m$. $\mathrm{KH}_{2} \mathrm{PO}_{4}$. Zur Stabilisierung des Enzyms ${ }^{13}$ wurden pro ml Homogenat $1,2 \mu \mathrm{g}$ TdR zugesetzt. Ein Teil des Homogenates wurde direkt getestet; ein anderer Teil wurde bei $105000 \mathrm{~g}$ (Spinco Model L, Rotor 50) zentrifugiert, und der Überstand zur Messung verwendet. Beide Fraktionen wurden ohne vorheriges Gefrieren sofort verarbeitet. $70 \mu \mathrm{l}$ Homogenat bzw. Úberstand wurden mit $50 \mu \mathrm{l}$ Inkubationsmedium bei $37^{\circ} \mathrm{C}$ inkubiert. Das Inkubationsmedium wurde in Anlehnung an Bollum und $\mathrm{V}_{\text {AN }}$ Potter ${ }^{14}$ hergestellt. $5 \mathrm{ml}$ dieses Mediums enthielten in $\mu \mathrm{Mol}$ : 400 Tris-HCl-Puffer $p_{\mathrm{H}} 7,6,240 \quad \mathrm{~K}_{2} \mathrm{HPO}_{4}, 160$ $\mathrm{MgSO}_{4}, 80$ ATP, $40 \mathrm{~m}$ TdR, 0,05 mC ${ }^{3} \mathrm{H}-\mathrm{TdR}$ (Radiochemical Center Amersham).

$\mathrm{K}_{2} \mathrm{HPO}_{4}$ wurde nach einem Vorschlag von KIELLEY $^{3}$ dem Inkubationsmedium zugesetzt; in Ansätzen ohne Phosphat war bei unserem Objekt die Enzymaktivität des Überstandes vermindert. Für die Blindproben wurden die Kinasen vor der $\mathrm{Zu}$ -

13 H. H. Hiatt u. T. B. Bojarski, Biochem. biophysic. Res. Commun. 2, 35 [1960].

14 F. J. Bollum u. R. Van Potter, Cancer Res. 19, 561 [1959]. gabe des Inkubationsmediums durch Erhitzen 2 min bei $100{ }^{\circ} \mathrm{C}$ inaktiviert. Nach 12,30 und $60 \mathrm{~min}$ wurden dem Inkubationsansatz Proben von jeweils $20 \mu \mathrm{l}$ entnommen, diese zu $30 \mu \mathrm{l}$ Aqua bidest. gegeben und $2 \mathrm{~min}$ bei $100{ }^{\circ} \mathrm{C}$ erhitzt. Das gefällte Protein wurde abzentrifugiert und ein aliquotes Volumen von $20 \mu \mathrm{l}$ für die chromatographische Trennung von TdR und den synthetisierten Thymidinphosphaten verwendet.

Chromatographie: Die von Furlong ${ }^{15}$ angegebene Methode zur Trennung von TdR und Thymidinphosphaten wurde nachgearbeitet. Hierzu wurden aliquote Mengen des Überstandes des Inkubationsansatzes auf $20.20 \mathrm{~mm}$ DEAE-Papierstücke aufgetragen, und der Anteil an nicht-phosphoryliertem TdR wurde mit 96-proz. Alkohol ausgewaschen. Da uns das von Furlong benutzte Papier (Reeve, Angel \& Co) nicht zur Verfügung stand, benutzten wir Whatman-Papier DE 20. Wir erhielten mit dieser Methode unbefriedigende Ergebnisse; TdR ließ sich nicht quantitativ herauswaschen. Auch Zusätze von unterschiedlichen Mengen einer $\left(\mathrm{NH}_{4}\right)_{2}-\mathrm{CO}_{3}$ Lösung zum Alkohol verbesserten das Ergebnis nicht.

Wir arbeiteten eine Trennungsmethode für aufsteigende Chromatographie mit Whatman-Papier DE 20 aus. Während die Laufmittel 96\%-, 50\%-, $30 \%$ Äthylalkohol, 2-m., 1-m., 0,5-m., 0,25-m., 0,1-m. $\left(\mathrm{NH}_{4}\right)_{2} \mathrm{CO}_{3}$-Lösung und 0,25-m. $\mathrm{NH}_{4} \mathrm{OOCH}-$ Lösung sich als unbefriedigend erwiesen, erreichten wir eine gute Trennung mit 0,005-m. $\left(\mathrm{NH}_{4}\right)_{2} \mathrm{CO}_{3}$ Lösung, wenn die Chromatographie mit diesem Laufmittel dreimal hintereinander wiederholt wurde. Bei diesem Verfahren wurden die Thymidinphosphate nicht getrennt; sie blieben in der Nähe der Startlinie sitzen. Der TdR-Spot stieg auf und erreichte beim dritten Lauf die Höhe der Lösungsmittelfront (Abb. 1). Das beschriebene Verfahren wurde für alle in dieser Arbeit beschriebenen Untersuchungen benutzt. $20 \mu \mathrm{l}$ vom Überstand des Inkubationsansatzes wurden auf die Startlinie aufgetragen. Eine gleich große aliquote Menge wurde auf Papierstücke von $10 \cdot 10 \mathrm{~mm}$ Größe gegeben, um die Gesamtaktivität zu messen.

Elution und Messung der Radioaktivität: Die Spots der Thymidinphosphate wurden in der in Abb. 1 angegebenen Weise ausgeschnitten und mit

15 N. B. Furlong, Analyt. Biochemistry [New York] 5, 515 [1963]. 


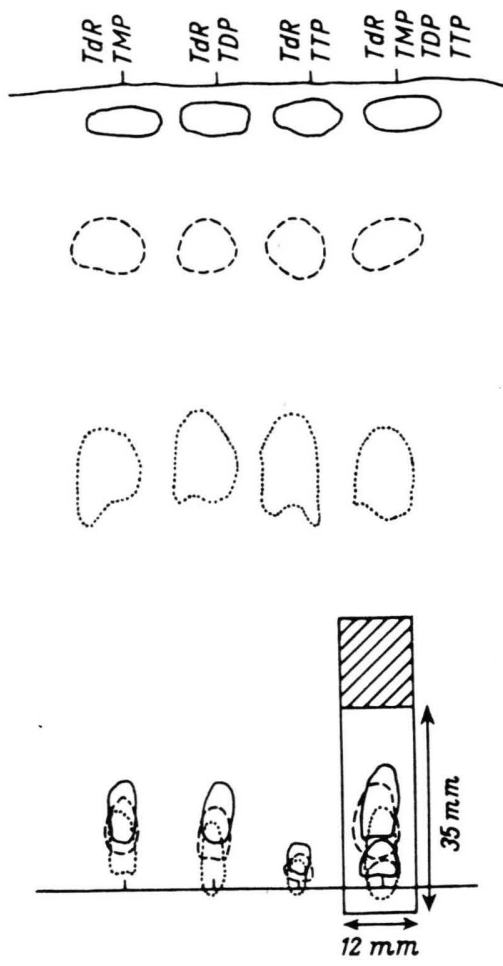

Abb. 1. Chromatographie von TdR, TMP, TDP und TTP in Gegenwart von Organextrakt auf Whatman-Papier DE 20. 1. Lauf $\cdots ; 2$. Lauf - - ; 3. Lauf —. Die umrahmte Fläche von $12 \cdot 35 \mathrm{~mm}$ wurde ausgeschnitten und eluiert. Die schraffierte Fläche diente zur Kontrolle. Die Lösungsmittelfront lag bei jedem Lauf 13,5 cm von der Startlinie entfernt.

$200 \mu \mathrm{l} \quad 0,5-m . \quad\left(\mathrm{NH}_{4}\right)_{2} \mathrm{CO}_{3} / 1-m . \quad \mathrm{NH}_{4} \mathrm{OH}-\mathrm{Lösung}$ eluiert. In der gleichen Weise wurden die Papierstücke zur Messung der Gesamtaktivität behandelt. Zur Kontrolle wurde auch der Bereich über dem Spot der Thymidinphosphate aus dem Chromatogramm ausgeschnitten. Es zeigte sich, daß die Impulsrate in diesem Bereich nur um die Größe des halben bis einfachen Nulleffektes erhöht war. Dem Eluat wurden $96 \mu \mathrm{l}$ entnommen und mit $10 \mu \mathrm{l}$ 0,25-proz. Gelatinelösung versetzt; $96 \mu \mathrm{l}$ dieser Mischung wurden auf nach Angaben von B elt z vorbehandelten Aluminiumschälchen aufgetragen und zwar in ein umschriebenes Areal derselben. Die Gelatinelösung gewährleistete ein gleichmäßiges Auftrocknen der Probelösung. Die Proben wurden 3 Stdn. im Trockenschrank bei $70^{\circ} \mathrm{C}$ aufbewahrt, um das $\left(\mathrm{NH}_{4}\right)_{2} \mathrm{CO}_{3}$-Salz zu vertreiben. Die Messung der Radioaktivität erfolgte mit einem Methandurch-

16 R. BeLtz, Analyt. Biochemistry [New York] 2, 303 [1961].

* Fräulein U. MAnNigel danken wir für ihre gewissenhafte Mitarbeit. flußzähler (FH 407) und einem automatischen Probenwechsler (FH 516). Von den gemessenen Impulsen wurden die der Blindprobe subtrahiert und die Syntheserate wurde in Prozent der Gesamtimpulse der Probe ausgedrückt ${ }^{*}$.

\section{Ergebnisse und Diskussion}

Das Ergebnis der Untersuchungen ist in Tab. 1 dargestellt. Es bestehen keine auffälligen Aktivitätsunterschiede zwischen Homogenat und Überstand. Die Aktivität der TdR-phosphorylierenden Kinasen ist in den unbefruchteten Eiern sehr hoch, sinkt nach der Befruchtung auf ein Drittel ab, bleibt in den befruchteten Eiern, den 4-Zellstadien und den mittleren Blastulastadien annähernd gleich und nimmt vom Blastulastadium an kontinuierlich bis zum Neurulastadium ab. Im Schwanzknospenstadium steigt die Aktivität und nimmt um ein Vielfaches im Larvenstadium zu. Die an verschiedenen Gelegen gemessenen Werte stimmen gut überein; größere Unterschiede wurden im 4-Zellstadium sowie beim Schwanzknospen- und Larvenstadium gefunden. Die Unterschiede der letzten beiden Stadien sind erklärlich, weil die Enzymaktivität hier stark ansteigt, und die exakte Bestimmung des Entwicklungsstadiums besonders bei Larven Schwierigkeiten bereitet.

Die Syntheserate zeigt im allgemeinen eine einfache geradlinige Abhängigkeit von der Inkubationszeit (Abb. 2). Nur in einigen Fällen ist die

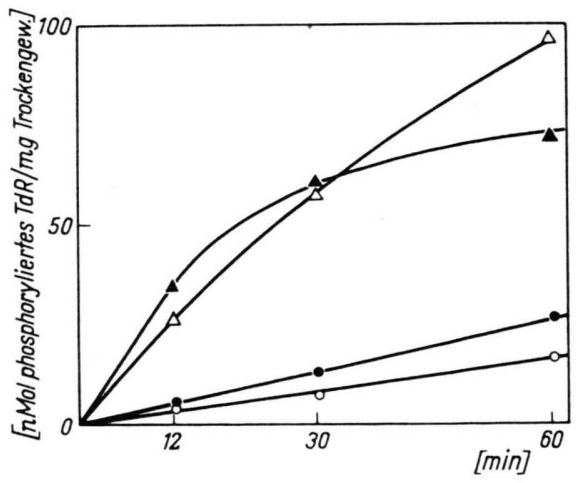

Abb. 2. Kinetik der enzymatischen Phosphorylierung von TdR. (a) Extrakte aus mittleren Gastrulastadien: Totalhomogenat - - - U Uberstand - O-O-; (b) Extrakte aus 8 Tage alten Larven: Totalhomogenat $-\boldsymbol{\Lambda}-\boldsymbol{\Delta}-$, Ủberstand $-\triangle-\triangle-$.

Kurve bei längeren Inkubationszeiten als 30 min gekrümmt. Für Larvenstadien erhält man Kurven mit starker Krümmung und zwar sind diese stärker ge- 


\begin{tabular}{|c|c|c|c|c|c|}
\hline \multirow{3}{*}{ Entwicklungsstadien } & \multicolumn{4}{|c|}{$\begin{array}{l}\text { Synthetisierte TdR-Phosphate } \\
\text { [nMol/mg Trockengewicht und min] }\end{array}$} & \multirow{3}{*}{$\begin{array}{c}\text { Mitoseindex } \\
\text { nach BRAGG } 22 \\
\text { bei Bufo cognatus }\end{array}$} \\
\hline & \multirow[b]{2}{*}{ Homogenat } & \multirow[b]{2}{*}{ Überstand } & \multicolumn{2}{|c|}{ Mittelwerte der Einzelmessungen } & \\
\hline & & & Homogenat & Überstand & \\
\hline unbefruchtete Eier & $\begin{array}{l}3,00 \\
2,50\end{array}$ & $\begin{array}{l}2,75 \\
2,63\end{array}$ & 2,75 & 2,69 & \\
\hline befruchtete Eier & $\begin{array}{l}1,40 \\
1,00 \\
0,90 \\
0,84\end{array}$ & $\begin{array}{l}0,88 \\
0,60 \\
0,66 \\
0,52\end{array}$ & 1,04 & 0,67 & \\
\hline 4-Zellstadien & $\begin{array}{l}2,06 \\
1,01 \\
0,29 \\
1,75\end{array}$ & $\begin{array}{l}1,17 \\
0,75 \\
0,27 \\
1,31\end{array}$ & 1,28 & 0,88 & \\
\hline mittlere Blastulae & $\begin{array}{l}1,39 \\
1,39\end{array}$ & $\begin{array}{l}1,36 \\
1,53\end{array}$ & 1,39 & 1,44 & 60,9 \\
\hline späte Blastulae & 0,77 & 0,50 & 0,77 & 0,50 & \\
\hline frühe Gastrulae & & & & & 55,4 \\
\hline mittlere Gastrulae & $\begin{array}{l}0,33 \\
0,42 \\
0,27\end{array}$ & $\begin{array}{l}0,26 \\
0,29\end{array}$ & 0,34 & 0,27 & 7,7 \\
\hline $\begin{array}{l}\text { Neurulae (Neural- } \\
\text { platte gebildet) }\end{array}$ & & & & & 7,4 \\
\hline $\begin{array}{l}\text { Neurulae Neural- } \\
\text { falten genähert) }\end{array}$ & $\begin{array}{l}0,24 \\
0,25 \\
0,25\end{array}$ & $\begin{array}{l}0,20 \\
0,17 \\
0,17\end{array}$ & 0,25 & 0,18 & \\
\hline $\begin{array}{l}\text { Neurulae (Neural- } \\
\text { rohr geschlossen) }\end{array}$ & & & & & 3,6 \\
\hline Schwanzknospen & $\begin{array}{l}0,68 \\
0,29 \\
0,63\end{array}$ & $\begin{array}{l}0,18 \\
0,26 \\
0,36\end{array}$ & 0,53 & 0,26 & \\
\hline Larven & $\begin{array}{l}3,48 \\
2,77\end{array}$ & $\begin{array}{l}4,29 \\
2,19\end{array}$ & 3,12 & 3,24 & \\
\hline
\end{tabular}

Tab. 1. Phosphorylierungsrate von TdR während der Entwicklung von Bufo bufo. Die Umsatzrate wurde aus den Zeit-UmsatzKurven entnommen. Bei krummlinigem Verlauf derselben wurde eine Tangente an den Kurvenanfangsteil gelegt.

krümmt bei Verwendung von Homogenaten als von Überstand.

Der ermittelte Aktivitätsverlauf der TdR-phosphorylierenden Kinasen während der Entwicklung des Krötenkeimes unterscheidet sich sehr deutlich von den entsprechenden Daten über andere Enzyme, vor allem solchen des intermediären Energiestoffwechsels. Es zeigte sich, daß eine Reihe von Enzymen, besonders die der Glykolyse und des Citronensäurecyclus während der Entwicklung vom befruch- teten Ei bis zum Abschluß der Neurulation eine nahezu konstante Aktivität aufweisen. Ein markanter Anstieg ihrer Aktivität setzt für gewöhnlich erst nahe dem Zeitpunkt des Ausschlüpfens der Larven ein ${ }^{17}, 18$. Zu Beginn der ersten Muskelkontraktionen beginnt nach Ergebnissen von Brown und Caston ${ }^{19}$ auch die Neubildung von Ribosomen und mit diesen der Anstieg im Gehalt an löslichen Proteinen. Diese Befunde wurden zwar an Rana pipiens erhoben, dürften aber für alle Amphibienarten gelten, da 
$\mathrm{ZEBE}^{20}$ die Aktivitätskonstanz an einer Reihe von Enzymen der Glykolyse und des Citronensäurecyclus vom Ei bis zur Neurula auch bei Bufo bufo verifizieren konnte.

Die Aktivität der TdR-phosphorylierenden Kinasen beginnt mit einem Maximum im reifen, unbefruchteten Ei. Der Aktivitätsabfall nach der Befruchtung läßt sich auf dem gegenwärtigen Stand unserer Kenntnisse über den DNS-Stoffwechsel im Amphibienei nicht erklären. Während der Furchungsteilungen bis zum mittleren Blastulastadium verbleibt die Aktivität der TdR-Kinasen auf einem mittleren Niveau. Dieser Entwicklungsabschnitt ist durch die Teilungsfreudigkeit der Kerne ausgezeichnet. Am Ende dieser Epoche sind die verschiedenen Plasmaareale des Keimes mit Zellkernen versehen und in Zellen aufgegliedert. Während der nun folgenden Gastrulation und Neurulation stehen Gestaltbildungsvorgänge im Vordergrund, die auf Verlagerung bestimmter Keimareale (Topogenese) und Wechselwirkung getrennter, nun in enge Nachbarschaft geratener Blasteme beruhen. Die Mitosehäufigkeit nimmt ab; entsprechend der biologischen Regel, daß in einem Organismus die Zellteilungsrate gering ist, während Differenzierungsprozesse ablaufen ${ }^{21}$. Der Mitoseindex sinkt bei Bufo cognatus nach Untersuchungen von BRAGG ${ }^{22}$ vom Gastrulationsbeginn bis zum Neurulastadium auf $1 / 8$ bis 1/15 des ursprünglichen Wertes ab (Tab. 1). Parallel damit fällt auch die Aktivität der TdR-phosphorylierenden Kinasen auf ein Minimum, wie aus Tab. 1 hervorgeht. Der nach durchlaufenem Schwanzknospenstadium wieder einsetzende steile Aktivitätsanstieg der TdR-Kinasen entspricht zeitlich dem nun beginnenden Wachstum und der Differenzierung der inzwischen ausgesonderten Organanlagen des Keimes, die sich biochemisch im Anstieg der Fraktion löslicher Proteine sowie im Aktivitätsgewinn zahlreicher Stoffwechselenzyme äußert.

Der Aktivitätsverlauf der TdR-Kinasen während der Frühentwicklung dürfte demnach die Mitosehäufigkeit im wachsenden Keim widerspiegeln. Ein

17 S. Løvtrup, C. R. Trav. Lab. Carlsberg, Sér. chim. 29, 261 [1955].

18 R. A. Wallace, Development Biol. 3, 486 [1961].

19 D. D. Brown u. D. Caston, Development Biol. 5, 4.12 [1962].

20 E. Zebe, unveröffentl., zit. bei F. Duspiva, 13. Colloq. Ges. physiol. Chem. 1963, p. 206.

21 N. J. Berril, Growth, Development and Pattern. W. H. Freeman \& Co., San Francisco and London 1961.

22 A. N. BraGG, Z. Zellforsch. mikroskop. Anatom. 28, 154 [1938]. klarer Zusammenhang mit der Neubildung von DNS ist hingegen nicht zu sehen. Wie quantitative Bestimmungen ${ }^{23-27}$ und histochemische Untersuchungen ${ }^{28}$ gezeigt haben, sind Amphibieneier mit einer ansehnlichen Reserve an cytoplasmatischer DNS ausgestattet. Eine Neusynthese von DNS findet bei Rana pipiens ${ }^{25}$ und Triturus-Arten ${ }^{26}$ erst im Blastulastadium statt und steigt kontinuierlich an. Bei Xenopus laevis wurde eine annähernd gleichmäßige Zunahme an DNS während der Entwicklung vom Ei bis zur freischwimmenden und Nahrung suchenden Larve beobachtet ${ }^{27}$.

Das Ergebnis dieser Arbeit steht also im Einklang mit der Erfahrung, daß teilungsfreudige Gewebe eine höhere Aktivität an TdR-Kinasen aufweisen als ruhende, adulte Gewebe. Da die Mitoserate in der animalen und vegetativen Hälfte der Blastula sowie in den verschiedenen Arealen der Gastrula verschieden ist ${ }^{22}$, wäre eine Erweiterung der am Ganzkeim erhobenen Befunde durch die Analyse der Aktivität von einzelnen Keimabschnitten sehr erwünscht. Die Entwicklung einer Ultramikromethode, welche die Analyse der TdR-Kinasen in so geringen Gewebemengen erlaubt, ist bereits in Angriff genommen.

Die Aktivität der TdR-Kinasen ist nur ein Glied im regulativen System der Kernteilung. Im Verlauf der Entwicklung des Keimes ändert sich die Kinetik der enzymatischen Phosphorylierung von TdR in charakteristischer Weise. Die stärkere Krümmung der Zeit-Umsatz-Kurven bei Verwendung von Homogenaten und Extrakten aus älteren Entwicklungsstadien (Abb. 2) deutet auf eine zunehmende Aktivität hemmender Faktoren. KIELLEY ${ }^{3}$ fand bei der Untersuchung der TMP-Kinase aus Leberhomogenaten eine stärkere Krümmung der Zeit-UmsatzKurven als bei der Verwendung von Tumoren, und führt den Unterschied auf Hemmfaktoren der Aktivität zurück, die in Lebergewebe, dessen Kerne in Teilungsruhe verweilen, besonders reichlich enthalten sind. Die zunehmende Krümmung der Zeit-Umsatz-Kurven von TdR-Kinasen aus Homogenaten

23 E. Hoff-Jörgensen u. E. Zeuthen, Nature [London] 169, 245 [1952].

24 L. C. Sze, J. exp. Zoology 122, 577 [1953].

25 J. R. GregG u. S. Løvtrup, Biol. Bull. 108, 29 [1955].

26 P. S. Chen, Exp. Cell. Res. 21, 523 [1960].

27 D. A. Bristow u. E. M. Deuchar, Exp. Cell. Res. 35, 580 [1964].

28 J. Brachet u. J. Quertier, Exp. Cell. Res. 32, 410 [1963]. 
und Extrakten älterer Keime dürfte demnach die prozentuale Zunahme ausdifferenzierter Zellen im Ganzkeim widerspiegeln. Die Natur der postulierten Hemmstoffe ist noch weitgehend unbekannt. KIELLEY ${ }^{2,3}$ vermutet, daß die rasch zunehmende Abflachung der Zeit-Umsatz-Kurve der TMP-Kinase aus Homogenaten von Lebergewebe durch TMP. spaltende Hydrolasen verursacht wird, die die Tumorgewebe fehlen. Ferner haben gewisse Reaktions- produkte der TMP-Kinasen (TTP) einen hemmenden Einfluß auf die TdR-Kinase der adulten und der regenerierenden Leber ${ }^{29}$.

Der Deutschen Forschungsgemeinschaft möchten wir für die großzügige Unterstützung der Arbeit unseren Dank aussprechen.

29 E. Bresnick, U. B. Thompson, H. P. Morris u. A. G. Liebelt, Biochem. biophysic. Res. Commun. 16, 278 [1964].

Studies on Organophosphorus Insecticides II

\title{
Distribution and metabolism of ${ }^{32} \mathrm{P}-\mathrm{O}$, O-dimethyl-2,2,2-trichloro-l-methoxyethyl phosphonate in the adult larva of "Prodenia litura F"
}

\author{
S. M. A. D. Zayed, A. Hassan and T. M. Hussein \\ Research Centre, Dokki, Cairo and Dept. of Biology, Atomic Energy Establishment, Cairo \\ (Z. Naturforschg. 20 b, 587-591 [1965] ; eingegangen am 28. Dezember 1964)
}

\begin{abstract}
The distribution of $\mathrm{P}^{32}$-labelled $O, O$-dimethyl-2,2,2-trichloro-1-methoxyethyl phosphonate (I) among different organs of adult larva of Prodenia litura F., showed that considerable radioactivity was translocated in hemolymph and gut. Significant accumulation of the $\mathrm{P}^{32}$-activity in the fat with time has been observed. The "in vivo" metabolic rate of I in Prodenia larvae was relatively small (12-16\% of the applied dose during 20 hours). The major metabolite $(75-85 \%)$ is produced by splitting of both $O$-methyl ester linkages and excreted as glucuronide. Monomethyl- $(5-10 \%)$ and dimethyl phosphates $(10-15 \%)$ were also identified as metabolic products.
\end{abstract}

The "in vitro" 1 and "in vivo" 2 metabolism of Dipterex in the adult larva of Prodenia litura F. has been recently studied. The relatively low toxicity of Dipterex to the cotton leaf worm has been attributed to its rapid detoxification ${ }^{2}$, as well as to the absence of cholinesterase in the larva ${ }^{3}$. $O, O$-dimethyl-2,2,2trichloro-1-methoxyethyl phosphanate I, obtained by methylation of Dipterex was found to possess a toxicity comparable to Dipterex ${ }^{4}$ towards Prodenia larvae. Moreover, recent studies have shown that I is much more stable than Dipterex and possesses a lower anticholinesterase activity ${ }^{5}$.

Therefore, it seemed of interest to study the metabolism of I in Prodenia larvae.

The present investigation is concerned with the distribution of I and its "in vivo" metabolism in the adult larva of the cotton leaf worm. For this study $\mathrm{P}^{32}$-labelled compound has been used.

1 S. M. A. D. Z ZaYed and A. Hassan, Canad. J. Biochem. [1965], in Press.

2 A. Hassan, S. M. A. D. Z Z (Submitted for publication).

\section{Material and Methods}

$\mathrm{P}^{32}$-labelled $O, O$-dimethyl-2,2,2-trichloro-methoxyethyl phosphonate I of specific activity $0.48 \mathrm{mC} / \mathrm{g}$. has been prepared by methylation of $\mathrm{P}^{32}$-Dipterex according to the method described by ZAYED et al. ${ }^{5}$.<smiles>COC(C(Cl)(Cl)Cl)P(=O)(OC)OC</smiles>

For the distribution and "in vivo" metabolism experiments, laboratory reared larvae $\left(5^{\text {th }}-6^{\text {th }}\right.$ instars $)$ of Prodenia litura F. were used after 3 hours starvation. Hemolymph has been used, for the "in vitro" metabolism studies.

\section{Distribution}

$50 \mu \mathrm{l}$ of an acetone solution containing $200 \mu \mathrm{g}$. $(16,000 \mathrm{cpm})$ of the $\mathrm{P}^{32}$-labelled compound were ap-

3 A. HAssan and S. M. A. D. Z Z AYED, Naturwissenschaften 52, 18 [1965].

4 Unpublished data.

5 S. M. A. D. Z Z AYED, A. HASSAN and I. M. I. FAKHR (Submitted to this journal). 